\section{Angular change in backscattering of light from the human lens with nuclear cataract}

\begin{abstract}
Purpose To determine how many meridians are needed to optimise detection of lens nuclear opacities in Scheimpflug photography utilising the Nidek EAS-1000 system.

Methods Ten eyes with nuclear cataract from 10 patients (age 53-83 years) were selected in this study. For each eye, the lens was imaged with the Nidek EAS-1000 system at the $0^{\circ}, 30^{\circ}$, $60^{\circ}, 90^{\circ}, 120^{\circ}$ and $150^{\circ}$ meridians, respectively. The intensity of the backscattering of light within a common lens nuclear area on the lens image was measured. In addition an aqueous suspension of polystyrene spheres was employed as a standard to evaluate the possible angular change caused by the camera system. Analysis of variance and the polynomial regression was used for data analysis.
\end{abstract}

Results The intensity of the backscattered light differs significantly among meridians. The highest intensity is found at the $90^{\circ}$ meridian. The intensity decreases towards the nasal and temporal side. There is also an angular variation caused by the camera system.

Conclusions There is a regular change in the backscattered light documented by the Nidek EAS-1000 system. It is sufficient to take one meridian to estimate the lens nuclear cataract. The variation in the backscattered light at different angles is caused partly by the variation of the camera.

Research Laboratory St Erik's Eye Hospital S-112 82 Stockholm Sweden

Tel: +468672 3011 Fax: +4686723352 e-mail: wen@brain.ste.ki.se Proprietary interest: None

This work was presented at ARVO, 1999

Received: 9 June 1999 Accepted in revised form: 26 August 1999 Scheimpflug photography has been used as an objective method for evaluation of the human lens nuclear density. ${ }^{1-5}$ In various clinical trials and research studies, Scheimpflug photography has proven its value. ${ }^{6-9}$
WEN QIAN

In Scheimpflug photography the slit beam can be rotated up to $180^{\circ}$ around the optical axis of the lens. This makes it possible to take images from any meridian of the lens. Currently, most studies use only one meridian for evaluation of the lens nuclear density. This is based on the assumption that the density within the human lens nucleus is homogeneously distributed. However, this assumption is not proven, and is questioned by some authors. ${ }^{3,10,11}$ If the nuclear density is not homogeneous, it is important to design an optimal measurement procedure.

In the study by Kashima et al. ${ }^{10}$ it was suggested that at least two meridians are needed to characterise the human lens nuclear cataract. In their study, 18 photographs for each lens from the $0^{\circ}$ to $180^{\circ}$ meridians, $10^{\circ}$ apart, were taken by a Topcon SL-45 camera. For each photograph, linear microdensitometry was performed through the centre of the lens with a measurement window of $0.44 \times 0.04 \mathrm{~mm}$. As a result, a one-dimensional profile was obtained for each photograph. The profile was used to determine the minimum number of photographs needed. However, it is not mentioned by Kashima et al. whether the lens cortex was included in the one-dimensional profile. The result could be affected if cortex is included because there is more variability of density within the cortex than the nucleus. ${ }^{13}$ Further, since the slit-beam in the Scheimpflug camera has a thickness of $0.08 \mathrm{~mm}$, the central volume of the nucleus was repeatedly measured when density was estimated from various angles. Consequently, the measurements were dependent.

In other studies, ${ }^{3,11}$ more than one meridian was used for estimation of lens nuclear density. However, it is not clear how many angles are needed for representation of the lens opacity.

The purpose of this study was to evaluate the angular change in backscattered light from the human lens with nuclear cataract. Based on the above information, the least number of angles required was estimated. 


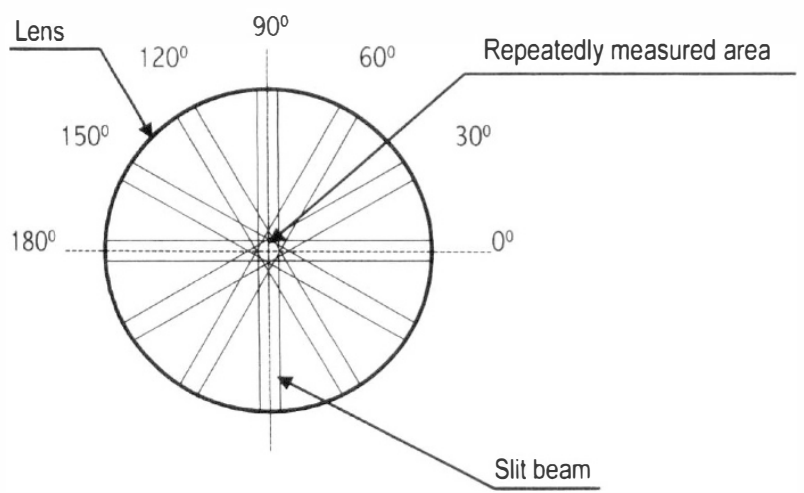

Fig. 1. Schematic drawing of the slit-beam at various angles within a human lens.

\section{Materials and methods}

In order to investigate the angular change of backscattered light from the lens nucleus, the largest possible number of angles should be measured. In the Nidek EAS-1000 system the slit-beam has a width of $0.08 \mathrm{~mm}$, according to the manufacturer (Nidek).

Therefore, when one estimates the density from different angles, the central volume of the nucleus is measured repeatedly (Fig. 1). The repeatedly measured volume increases with the increase in the number of angles investigated. In this study, the relationship between the number of angles and the repeatedly measured volume was evaluated (Fig. 2) as projected in the frontal plane. We decided to use six angles, which would give enough information about density distribution within a lens but a reasonably low overlap (10\%) volume with other angles (Fig. 2).

A total of 10 consecutive eyes with nuclear cataract from 10 patients ( 1 man, 9 women; age range 53-83 years) were included. The patients were referred to St Erik's Eye Hospital for cataract extraction and intraocular lens implantation. Informed consent was obtained from all patients.

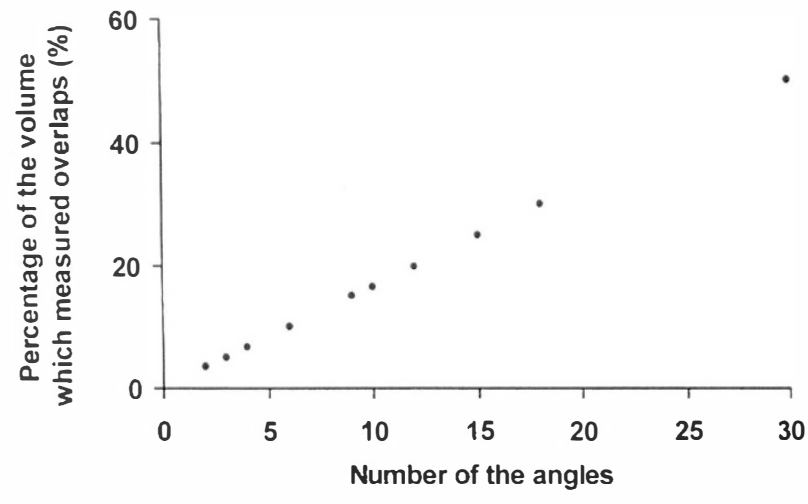

Fig. 2. Percentage of the volume which measured overlaps as a function of the number of angles investigated.

After dilating the pupils, each lens was imaged at the $0^{\circ}, 30^{\circ}, 60^{\circ}, 90^{\circ}, 120^{\circ}$ and $150^{\circ}$ meridians, respectively, by the Nidek EAS-1000 system (Fig. 1). All eyes were imaged with a slit length of $10.0 \mathrm{~mm}$. The intensity of the flash was selected according to the level of opacity. One image was taken for each meridian because in our pretest it was found that the variation is small between images within an angle $\left(9.07 \mathrm{cct}^{2}\right)$ compared with the variation among angles (130.47 $\left.\mathrm{cct}^{2}\right)$.

We have suggested a common lens nuclear area (CNA) as an optimal area for measurement of lens nuclear density. ${ }^{14}$ In this study, the CNA was employed for measurement of lens nuclear density (Fig. 3a).

Since the measurement area produced by the densitometric analysis program in the Nidek EAS-1000 system does not include CNA analysis, all lens images were transferred to another computer for image analysis. An algorithm for measurement of the intensity of backscattered light on the lens image was developed using the image processing software MATLAB (Math Works, USA). The mean intensity within the CNA was used to evaluate the angular change in backscattered light. Furthermore, to evaluate the possible local difference in intensity of backscattered light within the

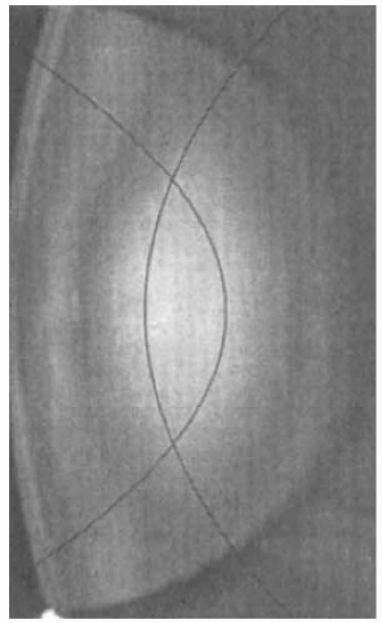

(a)

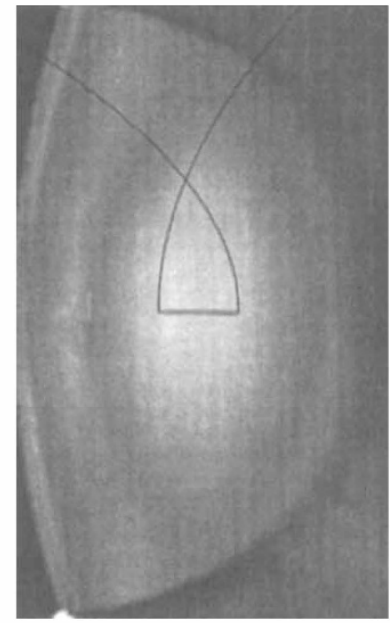

(b)

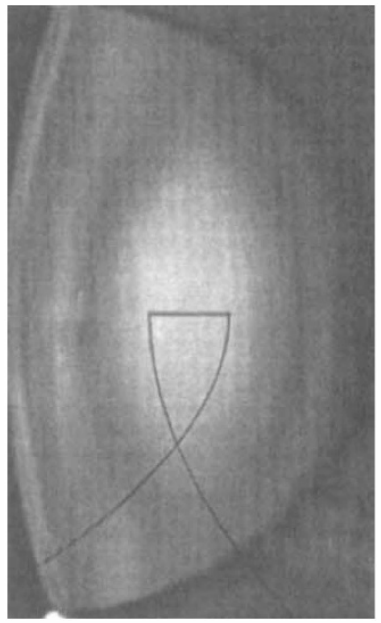

(c)

Fig. 3. (a) A common lens nuclear area (CNA) and its two parts, (b) and (c). 
Table 1. Analysis of variance of backscattered light from lens with nuclear cataract

\begin{tabular}{|c|c|c|c|c|}
\hline Source of variance & Degrees of freedom & Mean square (grey scale) ${ }^{2}$ & Expected mean square & Test statistic $\left(F_{f 1 ; f 2 ; 0.95}\right)$ \\
\hline Patients (P) & 9 & 7175.51 & $b c \sigma_{A}^{2}$ & \\
\hline Angles (A) & 5 & 372.71 & $c \sigma_{A \beta}^{2}+a c \kappa_{\beta}^{2}$ & $20.95(2.42)$ \\
\hline Locations (L) & 1 & 314.67 & $b \sigma_{A \gamma}^{2}+a b \kappa^{2} \gamma$ & $2.65(5.12)$ \\
\hline Int. (PA) & 45 & 17.79 & $c \sigma_{A \beta}^{2}$ & \\
\hline Int. (PL) & 9 & 67.62 & $b \sigma_{A \gamma}^{2}$ & \\
\hline Int. (AL) & 5 & 9.69 & $\sigma_{\varepsilon}^{2}+a \kappa_{\beta \gamma}^{2}$ & $1.10(2.42)$ \\
\hline Int. (PAL) + meas. & 45 & 8.77 & $\sigma_{\varepsilon}^{2}$ & \\
\hline
\end{tabular}

Int., abbreviation for the interaction factor; $a$, the number of patients $=10 ; b$, the number of angles for each eye $=6 ; c$, the number of locations at each angle $=2 ; \sigma^{2}$, the expected variance for the indexed random term; $A$, patients; $\varepsilon$, interaction among patients, angles and locations plus measurement error; $\kappa_{1}^{2}$ the factor corresponding to the variance for the index-fixed source; $\beta$, angles; $\gamma$, locations.

measured area, the CNA was divided into two parts (Fig. $3 b, c)$, and the mean density of each part in each meridian for each eye was analysed.

The possible variation in the angular change caused by the Nidek camera system was tested using a standard solution. In this study, an aqueous suspension of polystyrene spheres $(\mu=0.993 \mu \mathrm{m}$, standard deviation $=$ $0.010 \mu \mathrm{m}$; cat. no. 4009A and 4009B, Duke Scientific, USA) was used according to another study. ${ }^{12}$ An artificial anterior eye chamber was used to hold the aqueous suspension during photography.

Three different concentrations of the aqueous suspension, in the range corresponding to the density of the nuclear cataract in this study, were prepared by diluting the suspension with distilled water. The artificial chamber with the suspension was photographed with the Nidek EAS-1000 system by placing the chamber at the position corresponding to patient's eye. The slit length and flash light energy were set to $10 \mathrm{~mm}$ and $200 \mathrm{~W}$ s, respectively. Each concentration was imaged at the $0^{\circ}, 30^{\circ}, 60^{\circ}, 90^{\circ}, 120^{\circ}$ and $150^{\circ}$ meridians, respectively. Two images were taken for each meridian. The duration of image acquisition for each concentration was within $10 \mathrm{~min}$. The image of the suspension was analysed with MATLAB using a square $(1 \times 1 \mathrm{~mm})$ measuring area. ${ }^{12}$

The stability of the suspension over $10 \mathrm{~min}$ was estimated by taking images at regular intervals of $1 \mathrm{~min}$. The artificial eye chamber was filled with the diluted suspension, and photographed with the Nidek EAS-1000 system by putting the chamber at the position corresponding to the patient's eye. The image was analysed by MATLAB.

The data were analysed according to statistical models utilising analysis of variance (see Appendix). The pattern of the angular distribution of backscattered light was determined by polynomial regression. Considering the sample size, the significance level was set to 0.05 .

\section{Results}

To estimate the angular change of density within the lens with nuclear cataract, the mean density of each part (Fig. 3b, c) in each meridian for each eye was analysed. The results are given in Table 1 .
The intensity of backscattered light differs significantly among different angles in the lens nucleus (Table 1: Angles). The difference in intensity of backscattering of light between the parts of the CNA was not statistically significant (Table 1: Locations).

There is variation among different meridians caused by the Nidek camera (Table 2: Angles). The interaction between concentrations and the angles is insignificant (Table 2: Int. (CA)), which indicates that the pattern of the distribution of the intensity of backscattered light along angles is similar for different concentrations.

The suspension of polystyrene spheres was stable during image acquisition (Fig. 4). The coefficient of variance of the measurements from 10 images was $0.5 \%$.

Since the intensity of backscattering of light was different at different meridians for both the polystyrene suspension and the lens nucleus, the angular distribution of its intensity was further analysed.

For each concentration, the data from each angle were plotted as shown in Fig. 5, and fitted with a second-order polynomial. The continuous line in Fig. 5 shows the estimated pattern of distribution of the angular change.

For each lens image, the data from each of the six angles were fitted with a second-order polynomial and plotted (Fig. 6). The estimated distribution of the intensity of backscattering of light in the nuclear cataract is illustrated in Fig. 6 (continuous line) by substituting the mean of the regression coefficients of the 10 lenses examined into the second-order polynomial. Comparing Fig. 5 with Fig. 6, one can observe that the pattern of the angular distribution caused by the Nidek system is flatter but otherwise similar to the angular change in the

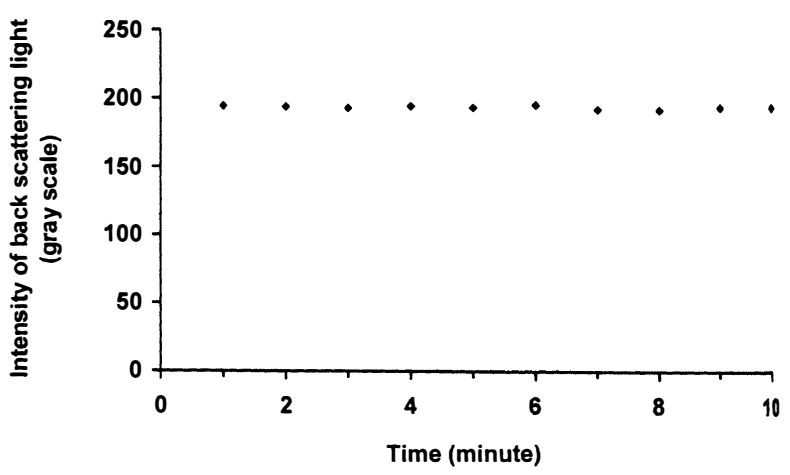

Fig. 4. Scatter plot of the intensity of backscattering light from 10 photographs with the same concentration of polystyrene suspension. 
Table 2. Analysis of variance of backscattered light from the suspension of polystyrene spheres

\begin{tabular}{lcccc}
\hline Source of variance & Degrees of freedom & Mean square (grey scale) & Expected mean square & Test statistic $\left(F_{f 1 ; f ; 0.95}\right)$ \\
\hline Concentration (C) & 2 & 5679.43 & $\sigma^{2}{ }_{\varepsilon}+b c \sigma^{2}{ }_{A}$ & \\
Angles (A) & 5 & 21.04 & $\sigma^{2}{ }_{\varepsilon}+c \sigma^{2}{ }_{A \beta}+a c \kappa^{2}{ }_{\beta}$ & $22.39(3.33)$ \\
Int. (CA) & 10 & 0.94 & $\sigma^{2}{ }_{\varepsilon}+c \sigma^{2}{ }_{A \beta}$ & $0.61(5.12)$ \\
Photos + meas. & 18 & 1.54 & $\sigma^{2}{ }_{\varepsilon}$ & \\
\hline
\end{tabular}

Int., abbreviation for the interaction factor; $a$, the number of concentrations $=3 ; b$, the number of angles for each concentration $=6 ; c$ the number of photographs at each angle $=2 ; \sigma^{2}$, the expected variance for the indexed random term; $A$, concentration; $\varepsilon$, the variance between photographs plus measurement error; $\kappa^{2}$ the factor corresponding to the variance for the indexed-fixed source; $\beta$, angles.

nucleus. Therefore, the angular change of the lens nuclear density can be partly explained by the variation caused by the Nidek camera system.

\section{Discussion}

In the present study we found that the intensity of backscattering of light varies with the angle (Table 1: Angles). The intensity of backscattering of light is highest at the $90^{\circ}$ meridian, and then decreases towards the horizontal meridian. To find the explanation of this density distribution, we tested the variation caused by the image acquisition instrument. The significant difference among angles (Table 2: Angles) indicates that there is a slight systematic variation in the Nidek camera system among angles, which also showed a peak at the $90^{\circ}$ meridian (Fig. 5). The cause of the variation is not known. As the curve of the angular change caused by the instrument (Fig. 5) is similar to that of the nucleus (Fig. 6), a part of the angular change in the nuclear density may be explained by the instrument.

During photography it was found that the lens image was affected by the eye position. For patients with a deeply located eye, the lens image at the $0^{\circ}$ meridian was darker since a part of the backscattered light was masked by the forehead and eyebrow. In order to minimise this interference, the chin rest was tilted up during photography. We also found that the lens images at meridian other than $90^{\circ}$ were always shadowed by eyelashes. The upper eyelid was lifted using a cotton swab to avoid the shadow. Care was taken not to press on the eyeball so that the lens image was not influenced by this manoeuvre. Considering the interference

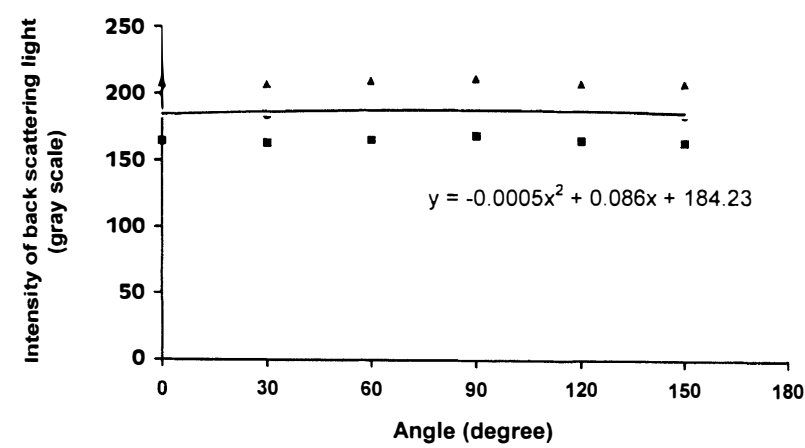

Fig. 5. Scatter plot of the angular change in backscattering light in three concentrations of polystyrene suspension. The continuous line is the estimated pattern of distribution of angular change. mentioned above, the angular change in the nuclear density could be attributed to the anatomical surrounding of the eye.

The finding of no significant difference in density between the two parts of the CNA within a meridian (Table 1: Locations) shows that the density within the section is homogeneously distributed. The finding of insignificant interaction between angles and locations (Table 1: Int. (AL)) indicated that there is no significant difference among angles considering the variation between parts of the CNA.

In Fig. 6 the intersect does not reflect the real level of the opacity in the example. In the Nidek EAS-1000 system the flash level of the light for imaging the lens can be adjusted from $50 \mathrm{~W}$ s to $200 \mathrm{~W}$ s for different degrees of opacity of the lens. The level of $200 \mathrm{Ws}$ is usually used for imaging a transparent lens and a lens with an early stage of cataract. For lenses with more dense cataract, the level of $150 \mathrm{~W}$ s or $100 \mathrm{~W}$ s should be adopted to avoid the image density reaching a saturation level. In the present study, the flash level was set $100 \mathrm{~W} \mathrm{~s}, 150 \mathrm{~W} \mathrm{~s}$ and $200 \mathrm{Ws}$, respectively, according to the degree of cataract.

In our previous study we found that the location and size of the lens nucleus varies among people. In order to optimise the possibility of detecting a change in nuclear density, the largest possible area in the nucleus should be measured. Meanwhile, the cortex should be excluded because of the variability in the cortex ${ }_{1}^{13}$ which will decrease the precision of the measurement of lens nuclear density. The CNA fulfils the above requirements. The CNA is determined based on an estimation of the tolerance limit for the boundary between nucleus and

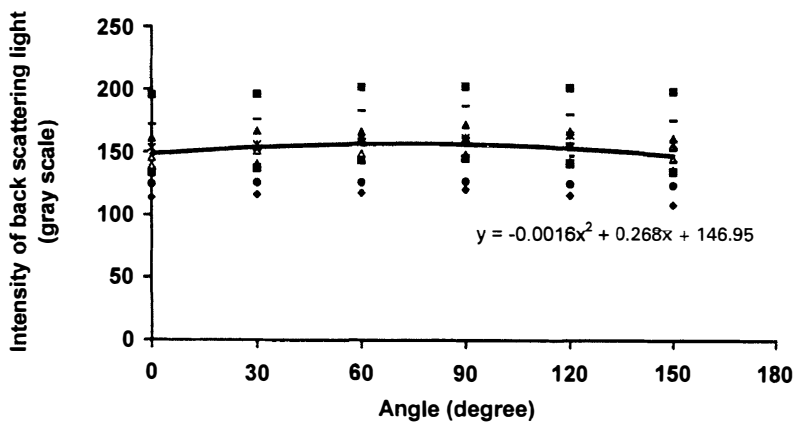

Fig. 6. Scatter plot of the angular change in backscattering light in 10 nuclear cataract lenses. The continuous line is the estimated pattern of distribution of angular change of light. 
anterior and posterior cortex. In this study the CNA was adopted for measurement of nuclear density on the lens image.

It is concluded that taking one image at the $90^{\circ}$ meridian is sufficient for evaluation of lens nuclear cataract.

\section{Appendix}

The angular change in backscattering of light in the lens with nuclear cataract

The angular change in backscattering of light among six meridians in the lens with nuclear cataract was analysed using an analysis of variance according to the following model: ${ }^{15}$

$\begin{aligned} x_{i j k}= & \mu+A_{i}+\beta_{j}+\gamma_{k}+(A \beta)_{i j}+(A \gamma)_{i k}+(\beta \gamma)_{j k}+(A \beta \gamma)_{i j k} \\ & +\varepsilon\left({ }_{i j k}\right)\end{aligned}$

Here, each value $x_{i j k}$ equals the sum of: the expected total mean, $\mu$; a term for the random variation among individuals, $A_{i}(i=1 \ldots a, a=10)$; a term for the fixed effect of angles, $\beta_{j}(j=1 \ldots b, b=6)$; a term for the fixed effect of locations, $\gamma_{k}(k=1 c, c=2)$; a term for interaction between individuals and angles, $(A \beta)_{i j}$; a term for interaction between individuals and locations, $(\beta \gamma)_{i k}$; a term for interaction between angles and locations, $(A \gamma)_{i k}$; a term for interaction among individuals, angles and locations, $(A \gamma)_{i k}$; and a term for random error of measurements, $\varepsilon(i j k)$.

\section{The angular change in backscattering of light in the polystyrene suspension}

The angular change in backscattering of light among six meridians for the polystyrene suspension was analysed using an analysis of variance according to the following model: ${ }^{15}$

$$
x_{i j k}=\mu+A_{i}+\beta_{j}+(A \beta)_{i j}+C_{k}(i j)+\varepsilon\left({ }_{i j k}\right)
$$

Here, each value $x_{i j k}$ equals the sum of: the expected total mean, $\mu$; a term for the random variation among concentration $A_{i}(i=1 \ldots a, a=3)$; a term for the fixed effect of angles, $\beta_{j}(j=1 \ldots b, b=6)$; a term for interaction between concentration and angles, $(A \beta)_{i j}$; a term for the random variation between photographs within angles and concentration, $C_{k}(i j)(k=1 \ldots n, n=2)$; and a term for random error of measurements, $\varepsilon(i j k)$.
The author would like to thank Professor Per Söderberg for comments on the manuscript and Associate Professor Enping Chen for help with the photography and comments on the manuscript. This study was supported by grants from the Stiftelsen Kronprinsessan Margaretas Arbetsnämnd för synskadade, St Eriks Ögonforskningsstiftelse.

\section{References}

1. Dragomirescu V, Hockwin O, Koch H, Sasaki K. Development of a new equipment for rotating slit image photography according to Scheimpflug's principle. Interdiscipl Topics Gerontol 1978;13:1-13.

2. World Health Organization. Guidelines for the clinical investigation of anticataract drugs in senile cataract. Copenhagen: WHO Regional Office for Europe, 1985.

3. Hockwin O, Dragomirescu, V, Laser H. Measurements of lens transparency or its disturbances by densitometric image analysis of Scheimpflug photography. Graefes Arch Clin Exp Ophthalmol 1982;219:255-62.

4. Chylack LT Jr, Rosner B, Cheng $\mathrm{H}$, et al. Sources of variance in the objective documentation of human cataractous change with Topcon SL-45 and Neitz-CTR retroillumination photography and computerised image analysis. Curr Eye Res 1987;6:1381-9.

5. Sasaki K, Sakamoto Y, Shibata T, et al. The multi-purpose camera: a new anterior eye segment analysis system. Ophthalmic Res 1990;22(Suppl 1):3-8.

6. Chylack LT Jr, McCarthy D, Khu P. Use of Topcon SL-45 Scheimpflug slit photography to measure longitudinal growth of nuclear cataracts in vivo. Lens Res 1988;5:83-93.

7. Datiles MB, Edwards PA, Trus BL, Green SB. In vivo studies on cataracts using the Scheimpflug slit lamp camera. Invest Ophthalmol Vis Sci 1987;28:1707-10.

8. Fujisawa K, Sasaki K. Changes in light scattering intensity of the transparent lenses of subjects selected from populationbased surveys depending on age: analysis through Scheimpflug images. Ophthalmic Res 1995;27:89-101.

9. Lasa MS, Datiles MB, Magno BV, Mahurkar A. Scheimpflug photography and post-cataract surgery posterior capsule opacification. Ophthalmic Surg 1995;26:110-13.

10. Kashima K, Unser M, Datiles M, Trus B, Edwards P. Minimum views required to characterise cataracts when using the Scheimpflug camera. Graefes Arch Clin Exp Ophthalmol 1993;231:687-91.

11. Wegner A, et al. Light scattering in the human lens in childhood and adolescence. Ophthalmic Res 1999;31:104-9.

12. Qian W, Söderberg PG, Chen E, Philipson B. A universal opacity standard for Scheimpflug photography. Ophthalmic Res, accepted.

13. Chen S, Chylack L, White O. Topcon SL-45 photography, a suitable technique for documenting nuclear but not cortical cataractous change in vivo. Invest Ophthalmol Vis Sci 1985;(Suppl)26:119

14. Qian W, Söderberg PG, Chen E, Magnius, K, Philipson B. A common lens nuclear area in Scheimpflug photographs. Eye 1993;7:799-804.

15. Snedecor GW, Cochran WG. Statistical methods. 7th ed. Ames, Iowa: Iowa State University Press, 1980:238-54. 